Copyright by the American Physical Society. Sears, A.; Batra, R. C., "Buckling of multiwalled carbon nanotubes under axial compression," Phys. Rev. B 73, 085410 DOI: http://dx.doi.org/10.1103/PhysRevB.73.085410

PHYSICAL REVIEW B 73, 085410 (2006)

\title{
Buckling of multiwalled carbon nanotubes under axial compression
}

\author{
A. Sears and R. C. Batra \\ Department of Engineering Science and Mechanics, M/C 0219, Virginia Polytechnic Institute and State University, \\ Blacksburg, Virginia 24061, USA
}

(Received 19 July 2005; revised manuscript received 19 December 2005; published 16 February 2006)

\begin{abstract}
Buckling of single-walled and multiwalled carbon nanotubes (SWNTs and MWNTs, respectively) due to axial compressive loads has been studied by molecular mechanics simulations, and results compared with those from the analysis of equivalent continuum structures using Euler buckling theory and the finite element method. It is found that a MWNT of large aspect ratio (length/diameter) buckles as a column with axial strain at buckling given reasonably well by the Euler buckling theory applied to the equivalent continuum structure. However, a MWNT of low aspect ratio buckles in shell wall buckling mode with the axial strain at buckling corresponding to the highest axial strain at buckling of one of its constituent SWNTs. A finite element model has been developed that simulates van der Waals forces by truss elements connecting nodes on adjacent walls of a MWNT; the axial strain at buckling from it is close to that obtained from the MM simulations but the two sets of mode shapes are different.
\end{abstract}

DOI: 10.1103/PhysRevB.73.085410

PACS number(s): 46.70.Lk

\section{INTRODUCTION}

It is now generally accepted ${ }^{1-7}$ that multiwalled carbon nanotubes (MWNTs) have mechanical properties quite different from those of single-walled carbon nanotubes (SWNTs). These differences are reflected in either their macroscopic material properties or their processing and handling behaviors or both. MWNTs are concentrically nested SWNTs with the distance between two adjacent walls of approximately $3.4 \AA$ Å. Molecular structures of a SWNT and a doublewalled nanotube (DWNT) are shown schematically in Fig. 1. Atoms on different walls of a MWNT do not share bonds with each other and interact mechanically through the weak van der Waals forces. This allows the walls of a MWNT to slide and twist freely relative to each other. When the outer wall of a MWNT is loaded in tension, it carries most of the load. Thus in tension a MWNT will have a lower specific stiffness than a SWNT. Yu et al. ${ }^{8}$ found that after the fracture of a MWNT, the inner walls remained intact and the broken outer wall slid freely in a "sword in sheath" manner. It suggests that the adjoining walls of a MWNT do not transmit tangential forces between each other. The wall-to-wall shear interaction is very weak as compared to the normal van der Walls forces. ${ }^{9}$

The van der Waals force experienced by atoms of two adjacent walls is normal to the walls and increases rather sharply with a decrease in the distance between the two walls; this effect can be seen in two ways. In bending deformations of a MWNT adjacent walls are seen to move in unison and act as a beam. The transmission electron microscope (TEM) images of bent nanotubes in composite materials show that the interwall distance remains relatively unchanged. When bent to high curvatures, the MWNTs are found to buckle locally into a rippling wave pattern throughout the length of the tubes. ${ }^{10,11}$ Another study in which a MWNT was repeatedly bent with a specially designed atomic force microscope (AFM) showed that these rippling patterns are reversible. ${ }^{12}$

The van der Waals force also comes into play in the axial compression of a MWNT due to caps at each end of the tube.
A TEM image of a MWNT end cap, taken from Yu et al. ${ }^{13}$ is shown in Fig. 2. The distance between adjacent walls of an end-cap equals nearly the ideal wall separation distance of $3.4 \AA$ A When compressed axially the endcaps may press together and transmit a part of the applied load to the inner walls. Thus a MWNT is expected to be stiffer in compression than in tension, e.g., see Thostenson and $\mathrm{Chou}^{14}$ who studied the buckling of a nanotube by first aligning the MWNTs through stretching, and then loading the composite containing the MWNTs in compression. Several buckling modes, columnar buckling, and kinking were found.

The failure mode of a nanotube in compression is different from that in tension. In tension, two failure modes, i.e., brittle failure due to the axial strain exceeding a critical value, and ductile failure due to excessive plastic deformations have been proposed. It has been suggested that these failure modes are best modeled by using quantum mechanics principles. ${ }^{15}$ However, in compression, buckling is the primary failure mode. In buckling, the bond structure of a nanotube does not change. This allows for the use of molecular mechanics (MM), molecular dynamics (MD), and continuum mechanics (CM) techniques to study compressive failure, and these three approaches have been used to analyze the buckling of SWNTs and MWNTs. In particular, Yakobson et $a l .{ }^{16}$ studied the buckling of a SWNT with the TersoffBrenner MM potential and a shell theory. Sears and Batra ${ }^{17}$ delineated the buckling of SWNTs by using the MM3 MM potential and the Donnell shell theory. Liew et al. ${ }^{7}$ have employed the MD technique to analyze buckling of SWNTs and
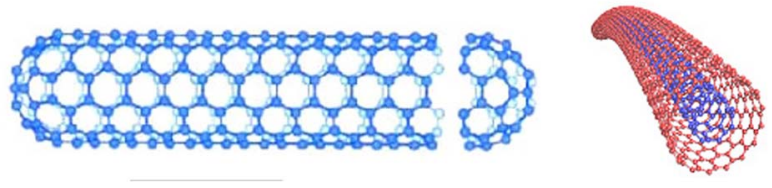

FIG. 1. (Color online) Left: Molecular structure of a SWNT (with end caps). Right: Molecular structure of a bent DWNT (without end caps). 

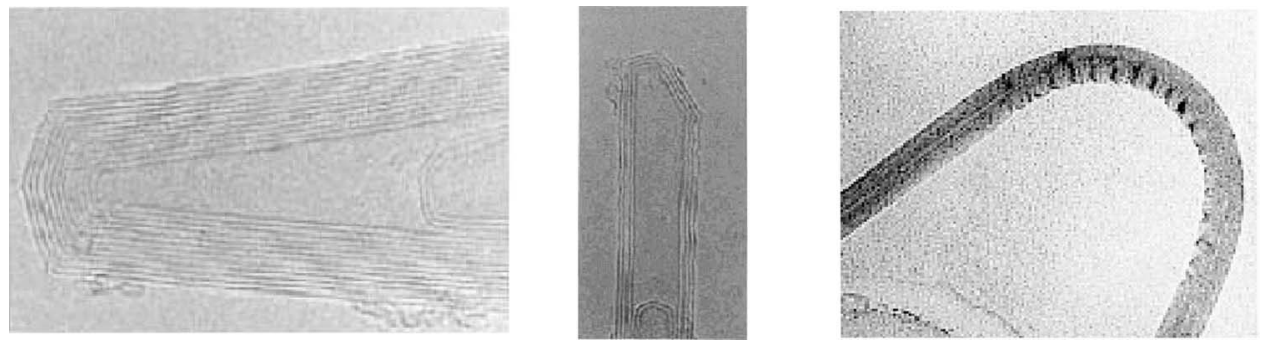

FIG. 2. TEM images of endcaps of a MWNT and the rippling pattern in a bent MWNT [Yu et al. (Ref. 13)].

MWNTs. Continuum structures equivalent to a SWNT employed in the above three works had different material and geometric parameters. Yakobson et al. ${ }^{16}$ assumed that the pi-bond thickness, $0.066 \AA$, is a good choice for the wall thickness. Liew et $a l^{7}$ found that the classical shell theory with the wall thickness of $1.54 \AA$ gave results in close agreement with their predictions of the buckling load from the MD simulations. Sears and Batra deduced the wall thickness of $1.34 \AA$ from MM simulations of the torsional and extensional deformations and the assumption of the material being isotropic and linear elastic for infinitesimal strains. Sears and Batra also employed the finite element method (FEM) to analyze three-dimensional deformations of the equivalent continuum structure.

During both columnar and wall buckling of a MWNT, the distance between any two of its adjacent walls may change thereby altering the van der Waals force among atoms on the two walls. However, it is not clear whether these interactions will delay the onset of buckling. $\mathrm{Ru}^{18}$ argued that the addition of an inner wall to a SWNT will decrease the axial strain at the onset of buckling of the structure. He used a CM approach and simulated the effect of van der Waals forces by applying a uniformly distributed pressure field on a wall; the pressure field was adjusted so as to give the same resultant force on each wall of the tube. He modeled each wall of a double-walled nanotube (DWNT) as a shell with bending stiffness $D(h)=E h^{3} /\left[12\left(1-\nu^{2}\right)\right]$, where $h$ is the wall thickness, $E$ Young's modulus, and $\nu$ Poisson's ratio.

Pantano et al. ${ }^{19}$ also modeled a DWNT as two concentric thin cylindrical shells each having $h=0.75 \AA, \nu=0.19, E$ $=4.84 \mathrm{TPa}$, and analyzed its buckling deformations by the FEM. The van der Waals force was simulated as equal and opposite pressures on the outer wall of the inner cylinder and the inner wall of the outer cylinder; the pressure varied with the distance between the two walls. For a SWNT deformed in bending, they found good correlation between the computed strain energies and the strain at buckling and/or kinking from the MM and the FE simulations. They also obtained reasonable agreement between the FE and the experimental results on the rippling of a MWNT deformed in bending. Pantano et al..$^{20}$ subsequently applied the same method to analyze buckling of a nine-walled nanotube in axial compression and found a rippling behavior that propagated through most of the walls towards the innermost tube. They also showed that the FE models gave better results for the buckling initiation and postbuckling behavior of thinner shells $(t=0.75 \AA)$ as compared to thicker walls $(t=3.4 \AA)$.

Arroyo and Belytschko ${ }^{21}$ have developed a FE model for MWNTs. Rather than using a thin shell theory, they replaced a tube wall by a membrane made of a hyperelastic material and deduced its mechanical properties directly from the Tersoff-Brenner MM potential, crystal elasticity, and a modified Cauchy-Born rule. The pressure equivalent to that exerted by the van der Waals forces was applied to the membrane. For torsional, bending and compressive deformations, they successfully reproduced local buckling, kinking, and rippling effects, which were very close to the shapes obtained by the MM simulations.

Here the buckling of MWNTs without endcaps is studied with the MM simulations, and also by analyzing threedimensional deformations of the equivalent continuum structure with the goal of elucidating the effect of van der Waals forces and the aspect ratio on columnar, shell-wall, and mixed-mode buckling. The nanotube structure is identified by using the White et al. ${ }^{22}$ notation, in which the graphite basal vector $(a, b)$ defines the cylindrical joining points of the nanotube. For MWNTs the notation $[(a, b),(c, d), \ldots]$, defines the structure of the innermost to the outermost tubes. The consideration of end caps introduces the difficulty of determining accurately the tube length, and also of finding an equivalent continuum structure. Previous studies ${ }^{18-20}$ on the buckling of nanotubes have also ignored endcaps. The actual tube length is larger than that considered here which means that buckling load will be a little smaller than that found here. Also, we have applied axial displacements to atoms that are $\sim 10 \AA$ from each end of the tube. In light of the SaintVenant principle ${ }^{23}$ which states that the precise prescription of boundary loads is unimportant provided that one considers deformations far away from the points of their application, our analysis provides reasonably good estimates of the buckling load. We note that experimentalists ${ }^{24-28}$ employ the Euler beam theory ${ }^{29}$ to interpret the response of a nanotube in bending, and extract Young's modulus.

\section{ANALYSIS OF THE PROBLEM}

\section{A. Molecular mechanics potential}

The MM3 class II pairwise potential with higher-order expansions, cross-terms, and type 2 (alkene) carbon atoms is
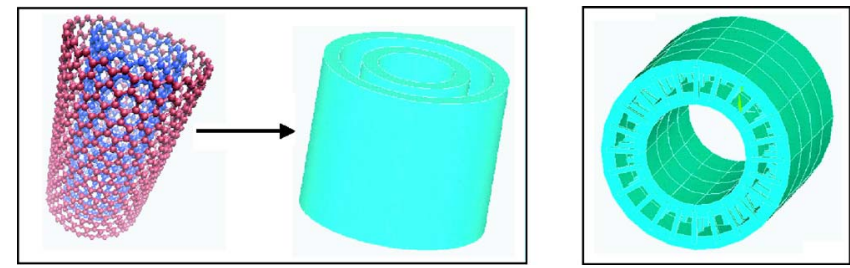

FIG. 3. (Color online) Left: a continuum structure equivalent to a DWNT. Right: a cross section of the FE model showing truss elements connecting nodes on two concentric tubes. 


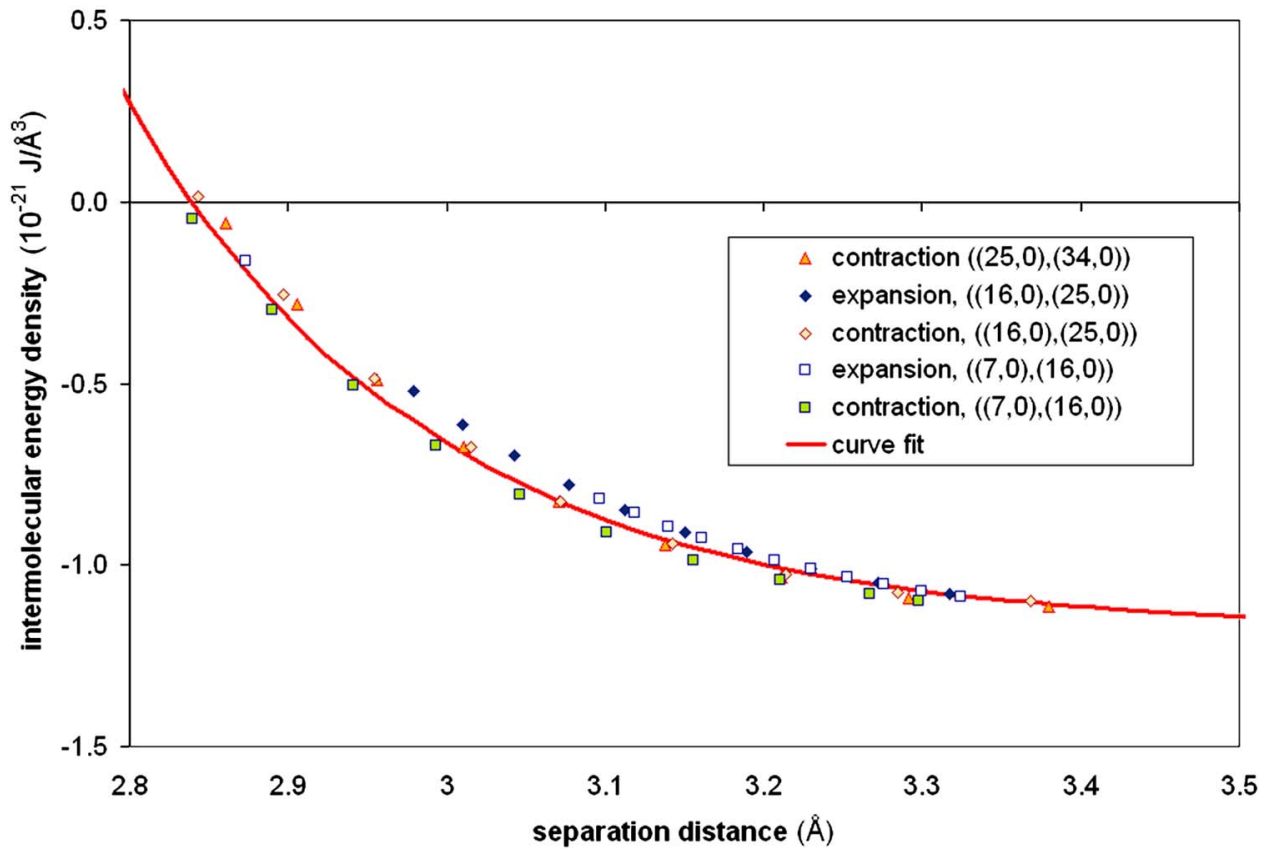

FIG. 4. (Color online) The van der Waals energy vs distance between two adjacent walls of a DWNT. used. ${ }^{30}$ This potential is appropriate for carbon nanotubes due to the similarity between graphitic bonds in a nanotube and the aromatic protein structures for which the potential was constructed.

\section{B. Virtual experiments}

The mechanical response of a nanotube is simulated with the computer code TINKER (Ref. 31 ) by following a procedure that closely mimics traditional macroscopic material tests. In summary, it involves finding the minimum energy configuration of an unloaded nanotube, referred to as the relaxed structure or the relaxed configuration. The deformed configuration is then estimated, displacement boundary conditions applied to atoms at the end faces to simulate axial compression, and remaining atoms moved axially to their estimated positions. The appropriate displacement boundary condition available in TINKER applies prescribed displacements to specified atoms in all three directions. In these simulations a circumferential ring of atoms $\sim 10 \AA$ from each end of the nanotube, on each wall, is chosen and displacement boundary conditions are enforced on these atoms. These boundary conditions are equivalent to clamped end conditions in an engineering mechanics analysis. With these atoms moved to their final positions and held there, the remaining atoms are allowed to move freely till the minimum energy configuration is attained. The process is repeated for different displacements prescribed on atoms on the circumferential ring to acquire data for the energy vs the axial strain curve. The axial strain is defined as the change in the initial length of the tube divided by the initial length. This duplicates what is done in experiments where the loading device moves end faces either at a prescribed rate or by a prescribed amount. The axial load is then usually read off from the machine. MM simulations were favored over MD simulations because they are quasistatic in nature as are the physical tests described above and the problems studied herein.
MM simulations neglect kinetic energy of the system, and essentially give one deformed state for a given strain level. An equilibrium configuration corresponds to the minimum of the potential energy. When displacements are prescribed at the end faces with the remaining surfaces free of surface tractions or loads, the equilibrium configuration corresponds to the minimum of the strain energy of the body.

In these studies the computer code TINKER automatically selected between the truncated Newton and the negative curvature methods of minimizing the potential energy. The convergence gradient and the cutoff distance were set at 0.001 r.m.s. and $15.0 \AA$, respectively. It should be noted that the use of the negative curvature method often overlooked the buckling of a tube. It was found in a previous study ${ }^{17}$ that a shell-wall buckled shape minimizes the strain energy of the tube by decreasing certain energy terms such as those due to a change in the bond length, while increasing others such as those due to the bond torsion and the out-of-plane terms.

A sinusoidal perturbation with an amplitude of $0.5 \%$ of the tube length is introduced in the relaxed configuration of the nanotube to facilitate its buckling. Prescribing a perturbation in the form of the estimated buckled shape is a common practice when analyzing buckling of nonlinear engineering structures. In a linear theory, such as the Euler beam theory, ${ }^{29}$ the buckling problem is analyzed as a stability event yielding only the critical buckling load, and the amplitude of the buckled shape is infinity; it thus precludes the stable growth of a buckled mode shape. In a nonlinear theory a perturbation on the perfect geometry is required to allow for the growth of the mode shape. Thus a separate criterion such as the lateral maximum deflection is required to define the initiation of the buckling of a structure. The estimated buckled shape, often found from the linear analysis, generally allows for the smooth growth of the buckled shape. Random noiselike perturbations used in nonlinear $\mathrm{FE}$ analyses ${ }^{32}$ have been found to work very well. Their advantage is that they do not favor a buckling mode. However, they some- 


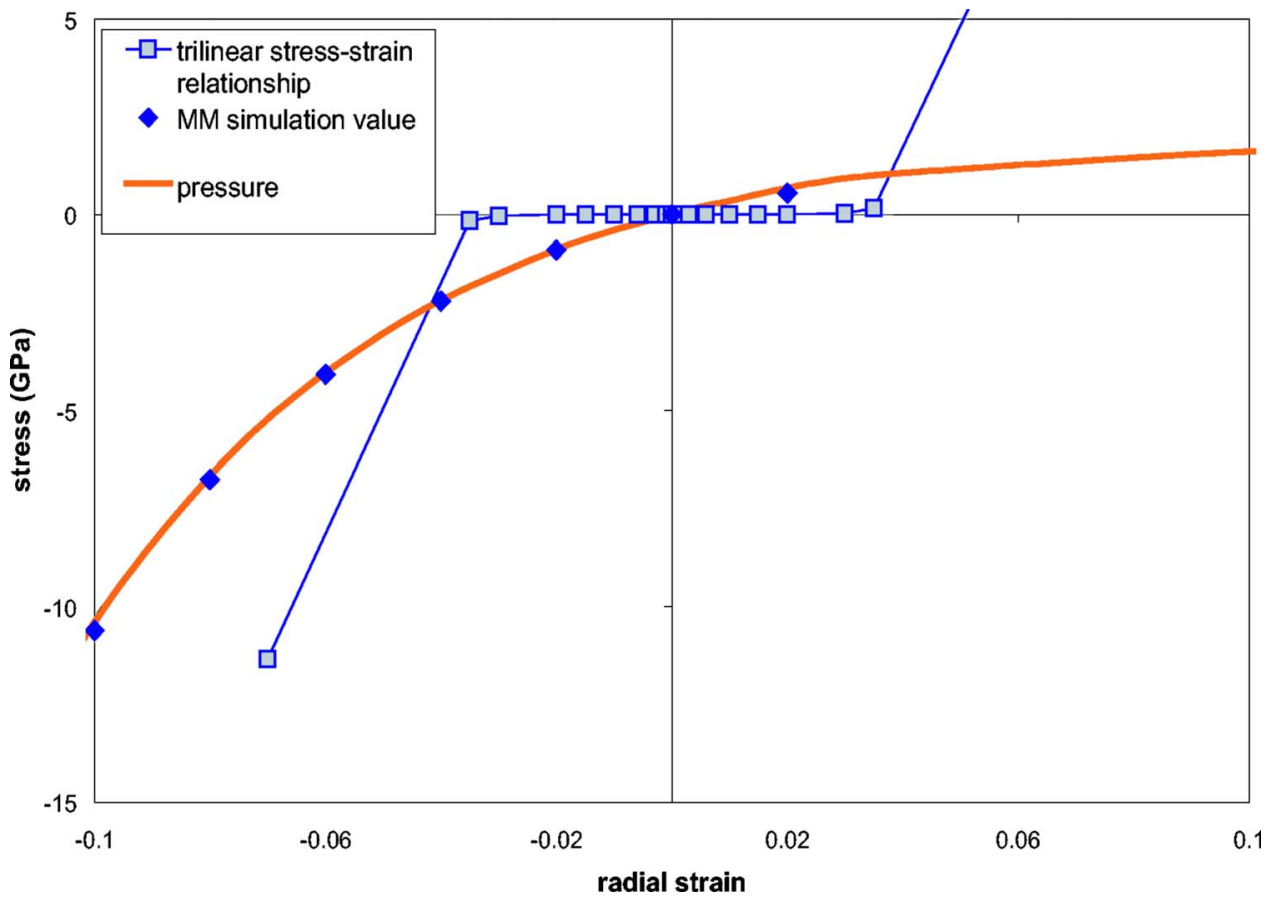

FIG. 5. (Color online) Radial pressure vs radial strain derived from MM simulations of radial expansion/contraction of a wall of a nanotube.

times inhibit the growth of a buckling mode and must be performed numerous times to insure that the lowest critical buckling load or strain has been found. Such perturbations were attempted in this study and were not found to perform well. While buckling load and strain is sensitive to the perturbation size, the effect can be minimized through convergence studies and the smallest acceptable amplitude of the perturbation can be ascertained. The tube is assumed to have buckled when the maximum lateral deflection equals the smaller of $1 \%$ of its length or $10 \%$ of the radius of the $(16,0)$ SWNT; the former criterion was used for long tubes which buckled in columnar modes, and the latter for the short tubes which buckled in shell-wall buckling modes.

\section{Continuum models}

Following the work of Sears and Batra, ${ }^{17}$ we modeled a SWNT as a cylindrical tube made of an isotropic linear elastic material with the following values of material and geometric parameters $h=1.34 \AA, E=2.53 \mathrm{TPa}$, and $\nu=0.19$. The equivalent continuum model of a MWNT is comprised of concentric tubes of mean radius equal to that of a wall of a MWNT, $h=1.34 \AA, E=2.53 \mathrm{TPa}$, and $\nu=0.19$. While analyzing it as an Euler column or beam, ${ }^{29}$ it was assumed that the distance between two adjacent walls remains unchanged; it is referred to as a rigid gap model signifying a fixed gap between two adjacent walls. This assumption allows the MWNT column to have an area and moment of inertia which

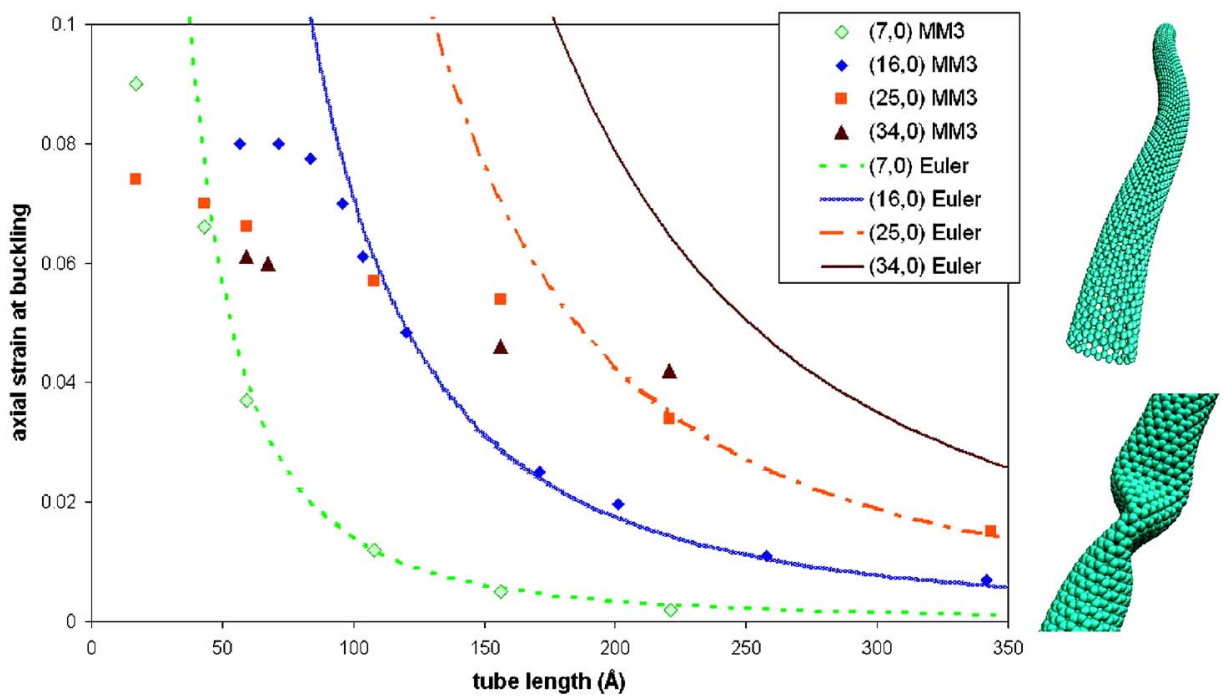

FIG. 6. (Color online) The axial strain at the onset of buckling vs the tube length for various SWNTs (two examples of buckling mode shapes are also shown; top: columnar buckling, bottom: shell wall buckling). 

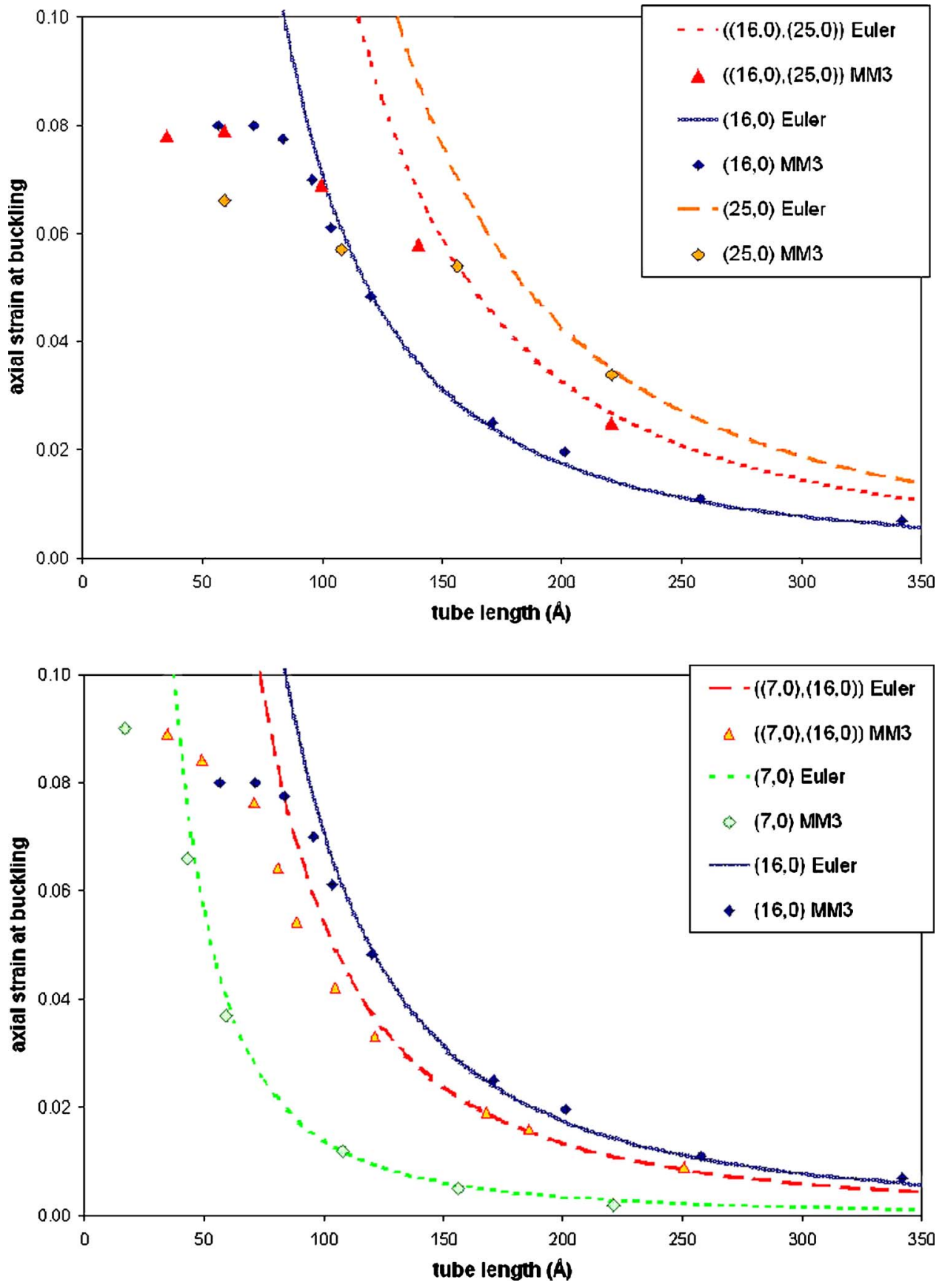

FIG. 7. (Color online) Axial strain at buckling for two differently structured DWNTs and their constituent SWNTs vs the tube length; (a) top $[(16,0),(25,0)]$, (b) bottom $[(7,0),(16,0)]$. are simply the sum of those for the individual walls. We note that the Euler buckling theory can only predict the critical buckling strain for columnar buckling. Shell-wall buckling modes were found by modeling each tube as a shell, and analyzing deformations of the structure with the FEM using eight-node quadratic shell elements. The problem formulation allowed for small strains but large rotations.

Numerical experiments with different FE meshes revealed that the computed solution for a $(16,0)$ SWNT of aspect ratio (length/diameter) 1:1 converged when 16 elements were employed in the circumferential direction; the convergence criterion was defined as the coarsest mesh for which the axial strain at the onset of buckling became independent of the mesh size. The effect of van der Waals forces is accounted for by incorporating uniform truss elements connecting nodes on adjacent cylindrical shells. Nodes on adjoining cylindrical shells were taken to lie on the same radial lines allowing for a regular distribution of uniform radial truss elements as depicted in Fig. 3. The area of cross section $A$ of a truss element was chosen so that $(\mathrm{A} \times$ number of truss elements/axial length of the cylinder) $=\Pi\left(D_{2}-D_{1}\right)$, where $D_{1}$ and $D_{2}$ are the mean diameters of the inner and the outer walls of the DWNT. Due to a mismatch in the number of degrees of freedom of a node on a cylindrical shell and that at the ends of a truss, a set of coincident nodes on virtual elements were defined; a virtual element linked a node on a truss to its counterpart on the cylindrical shell.

Young's modulus of a truss element was assumed to vary with the axial strain in that element. Two such relations were 
developed and used. The first one is called the pseudopressure model and is based on MM simulations of radial expansion/contraction of a wall of a DWNT. ${ }^{33}$ A relation between the pressure acting on a wall and its radial displacement is developed from the van der Waals intermolecular energy versus the wall separation distance depicted in Fig. 4. Equation (1) describes the least squares fit to the intermolecular energy $W$ vs gap $s$ between two adjacent walls

$$
W=2 \pi L \frac{K\left[b\left(s+r_{i}\right)+1\right]}{b^{2}\left(r_{o}^{2}-r_{i}^{2}\right)} \exp [-b(s-c)],
$$

where $2.6 \AA<s<3.4 \AA, K=9.5 \times 10^{-22} \mathrm{~J} / \AA^{3}, b=5.31 / \AA, c$ $=3.52 \AA$. The radial force versus $s$ relation can be found by differentiating Eq. (1) with respect to $s$ from which pressure can be computed. The resulting pressure versus the radial strain relation is shown in Fig. 5. It was used to deduce the axial force versus the axial displacement relation for a truss element, and is called the "pseudopressure model." Since the sum of the area of cross section of truss elements was set equal to the average surface area of the walls, the radial pressure equals the axial stress in a truss element.

Analysis of buckling deformations with this pressure versus strain relation gave axial strains at buckling that were higher than those obtained from the MM simulations. Thus a second axial force versus axial displacement relation, termed the "trilinear truss model," was developed. It has relatively low modulus near the ideal wall separation distance or zero radial strain, and allows the adjoining walls to move nearly independently of each other and small perturbations to develop. The modulus is nearly constant for moderate values of the axial strain in a truss element, and then increases very rapidly to a value much higher than that given by Eq. (1) so as to force the two adjoining walls to move in unison with each other. We emphasize that this ad hoc model is not a curve fit, and is based on an intuitive argument that the two tubes deform essentially independently in the beginning, and after certain level of straining their deformations are coupled to each other. To closely mimic the MM simulations, the initial configuration of the continuum structure was given the same perturbation as the molecular structure, and nodes at the end faces of the continuum structure were moved axially and then kept fixed in all three directions.

\section{RESULTS}

\section{A. Buckling of a SWNT}

The buckling of a SWNT does not involve interactions between adjacent walls; one can thus examine the adequacy of the equivalent continuum structure. The axial strain at buckling versus the length of a $(16,0)$ tube (tube diameter, $D=11.9 \AA$ ) for various values of the tube length is shown in Fig. 6. The MM simulation results are compared with those obtained from the Euler column theory and the FE analyses of continuum cylindrical tubes of different wall thicknesses but the same axial stiffness, i.e., having the same value of $\mathrm{E}$ multiplied by the area of cross section. For tube lengths greater than $120 \AA(L / D>10)$, the three sets of results are very close to each other. However, for tube lengths less than

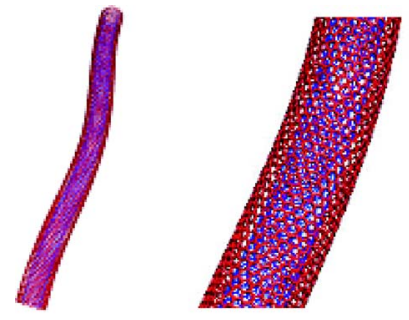

(a)

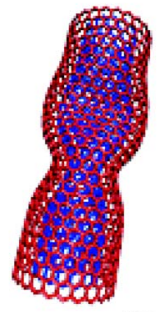

(b)

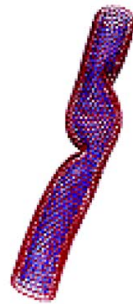

(c)
FIG. 8. (Color online) Examples of MWNT buckling modes (a) columnar with close-up view, (b) shell wall, (c) crimped.

$100 \AA$ the axial strains computed from the MM simulations and the FE analysis are very close to each other but both are much less than that given by the Euler buckling theory. This is because the buckling mode shifts from a columnar mode to a shell wall mode for the MM and the FE simulations which the Euler theory cannot predict.

\section{B. Correlation between the buckling of SWNTs and MWNTs}

A close scrutiny of results for the buckling of a SWNT suggests the following question: will the axial strain at buckling of a MWNT equal the lowest of those at which SWNTs constituting it buckle or will the adjacent walls tend to support each other and increase the axial strain at buckling? One way to answer it is to compare results of buckling of a double wall nanotube (DWNT) with those of buckling of its SWNTs; these are shown in Figs. $7(\mathrm{a})$ and $7(\mathrm{~b})$ for the $[(7,0),(16,0)]$ and the $[(16,0),(25,0)]$ DWNTs. These DWNTs were chosen because the wall separation distance in MWNTs produced from them is close to $3.4 \AA$. Results for the constituent SWNTs are also shown for easy comparison.

For large aspect ratios (length/outer wall diameter) of the $[(16,0),(25,0)]$ DWNT, MM simulations reveal that the axial strain at buckling of a DWNT is between those of its constituent SWNTs, and for each tube the MM simulation result is close to that predicted by the Euler buckling theory. This implies that the walls act in unison, which is confirmed by the columnar buckling mode shape that retains the ideal wall separation distance between the two walls as seen in Fig. 8 . The wall separation distance was found to remain near $3.4 \AA$ throughout the postbuckling response. It supports the rigid gap assumption made in the analysis of continuum structures equivalent to MWNTs with the Euler buckling theory. However, for short tubes the axial strain at buckling of the DWNT equals that of the $(7,0)$ SWNT. In this range, the $(25$, $0)$ SWNT due to its shallower curvature buckles in the shell wall buckling mode (e.g., see Fig. 8) at a lower value of the axial strain than that needed for it to buckle in the axial buckling mode. Thus, one might expect the outer wall of the DWNT to begin to buckle first and cause the DWNT to buckle. However, the inner wall with its higher value of the axial strain in shell-wall buckling mode remains straight and supports the outer tube until the axial strain reaches that of the inner $(16,0)$ tube to buckle in shell-wall buckling mode and both walls of the DWNT buckle in unison in a shell-wall buckling mode. The situation is less clear in the transition 


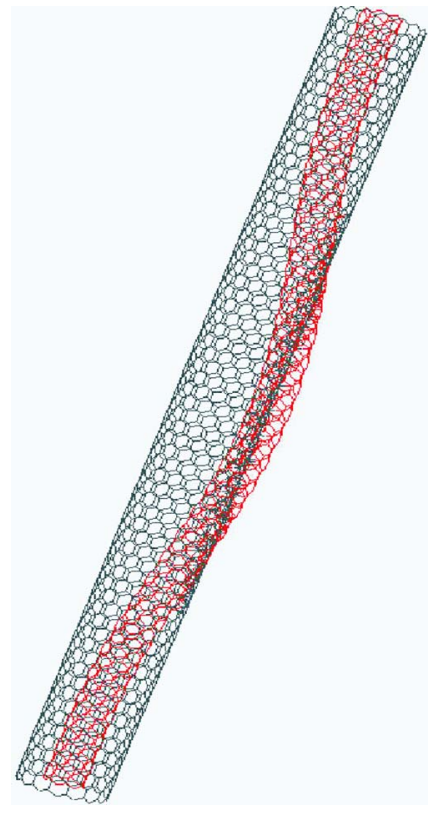

FIG. 9. (Color online) Buckling of a $[(7,0), 16,0)]$ DWNT in the absence of van der Waals forces.

zone where mixed column and crimping buckling modes are prevalent. The same trends are present for the $[(7,0),(16,0)]$ DWNT and the axial strain at buckling is intermediate between those of the constituent SWNTs for large aspect ratios but equals that of the $(16,0)$ SWNT for small aspect ratios.

In order to clearly delineate the effect of van der Waals forces we also analyzed the buckling of a $[(7,0), 16,0)]$ DWNT by artificially annulling these forces; the resulting buckled shape is exhibited in Fig. 9. It is clear that the inner tube buckled first and passed through the walls of the outer tube. With the effect of van der Waals forces included, the outer tube would have prevented the inner tube from buckling. This would occur until the axial strain in the two tubes reached a level at which they will buckle into a columnar mode shape.

For the DWNT replaced by an equivalent continuum structure with the distance between the two cylindrical tubes kept fixed, predictions from the Euler buckling theory match with those from MM simulations for tubes of large length but deviate considerably for tubes of small length due to the shell wall buckling experienced by tubes of small length. The deviation between the axial strains at the initiation of buckling from MM simulations and the Euler buckling theory occurs at a smaller value of length for a DWNT than that for a SWNT.

Buckling of a $[(7,0),(16,0),(25,0)]$ TWNT was also studied by both MM simulations and the Euler buckling theory. The axial strain at the initiation of buckling versus the tube length is plotted in Fig. 10. As for the SWNTs and the DWNTs the axial strain at the initiation of buckling in long tubes matches well with that computed from the Euler theory and they have columnar buckling modes. With a decrease in the tube length, the axial strain at buckling begins to deviate from that given by the Euler theory and the buckling mode transitions from pure columnar to columnar with crimps. For tube lengths smaller than $100 \AA$, pure axial shell wall buckling modes emerge at the axial strain close to that of the $(16,0)$ SWNT. For a fixed length, the $(16,0)$ SWNT has the highest axial strain at buckling because the $(7,0)$ SWNT buckles in the columnar mode at a much lower axial strain. A close scrutiny of results depicted in Figs. 8 and 10 reveals that the axial strain at buckling of a low aspect ratio MWNT can be reasonably predicted from the highest axial strain at buckling of any one of its constituent SWNTs.

Some interesting buckling modes found from the MM simulations for a $[(16,0),(25,0),(34,0)]$ TWNT are shown in Fig. 11. For a moderate tube length of $156 \AA(L / D=\sim 6)$ the outer tube is first seen to buckle into rippling pattern at $7 \%$ axial strain. This rippling pattern is similar to buckling patterns found in bent MWNTs where multiple shallow shell

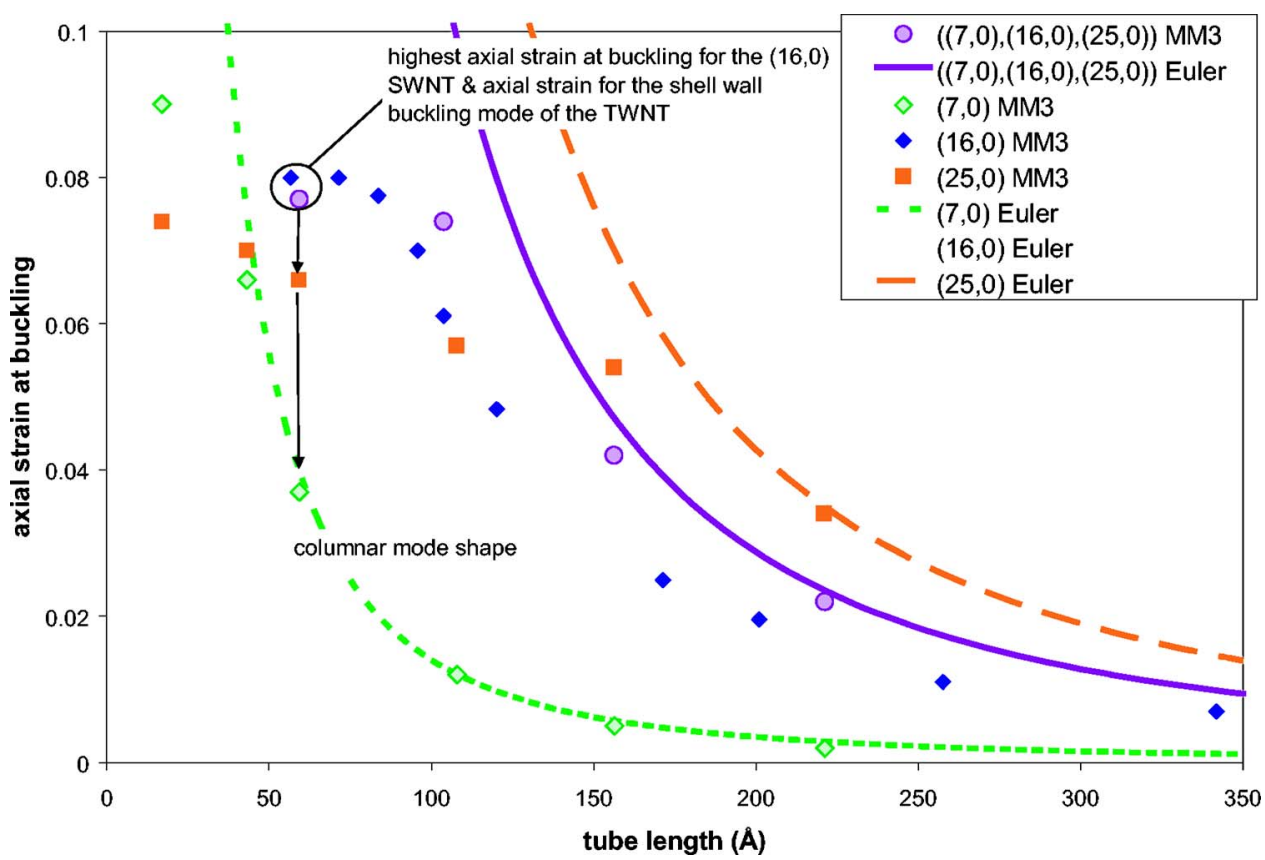

FIG. 10. (Color online) Axial strain at buckling vs the tube length for a TWNT and its constituent SWNTs. 


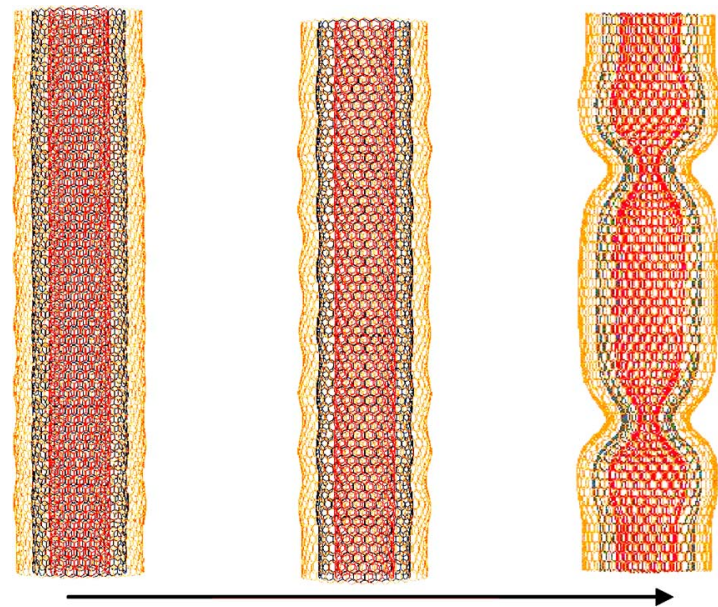

increasing compressive strain

FIG. 11. (Color online) The emergence of rippling waves in a TWNT followed by deep shell wall buckled shape with increasing axial compressive strain.

waves with equivalent wavelengths are repeated. However, here the buckling is caused by axial compression, and ripples go only one wall deep. As the tube is compressed further, the middle tube is also forced into the rippling pattern at $7.9 \%$ axial strain. Shortly thereafter at $8.0 \%$ axial strain the whole tube buckles into the shell wall buckling mode seen on the right. This phenomenon reinforces the hypothesis that the inner walls effectively support the outer walls against buckling. For a different initial perturbation the same tube is seen to buckle into a fully developed shell wall buckling mode at $8 \%$ strain; thus the buckling response is dependent upon the initial perturbation and is nonunique. However, typical values of the axial strain for the shell wall buckling mode of a $(16,0)$ SWNT are about $8.0 \%$.

\section{Results from the continuum model of a MWNT}

As the buckling of a SWNT does not involve interactions between adjacent walls, the adequacy of the equivalent continuum structure of a SWNT can be studied. The axial strain at buckling vs the length of a $(16,0)$ tube (tube diameter, $D$ $=11.9 \AA$ ) for various values of the tube length is shown in Fig. 12. The MM simulation results are compared with those obtained from the Euler buckling theory, and the analyses of equivalent continuum cylindrical tubes by the FEM with the tube modeled as a shell. For tube lengths greater than $120 \AA$ $(L / D>10)$, the three sets of results are very close to each other, indicating that both the Euler buckling theory and the FE analysis of the equivalent continuum structures accurately predict global buckling modes. For short tubes in the shell wall buckling regime, the MM simulations yield the axial strain at buckling versus tube length curve that is nearly flat around the $8.0 \%$ axial strain. In this range, the FE analysis predicts the correct mode shape, with a somewhat flat curve with axial strain at buckling between 8.2 and $8.6 \%$. This shows the adequacy of the equivalent continuum structure of a SWNT and the analysis techniques.

Previous studies ${ }^{28}$ on bending of a continuum structure equivalent to a DWNT showed that when the gap between two concentric cylindrical tubes is assumed not to change, the Euler beam theory gives results quite close to those obtained from MM simulations. We note that MM simulations showed that the gap between two adjacent walls does not change during bending. Furthermore, the Euler buckling theory applied to a continuum structure equivalent to a SWNT and a MWNT of large aspect ratios predicts axial strain at buckling very close to that given by the MM simulations. Therefore, analyses of buckling deformations of a MWNT should focus on local buckling effects and on the transition between global and local effects. Deformations of a $[(16,0),(25,0)]$ DWNT and a $[(16,0),(25,0),(34,0)]$ TWNT

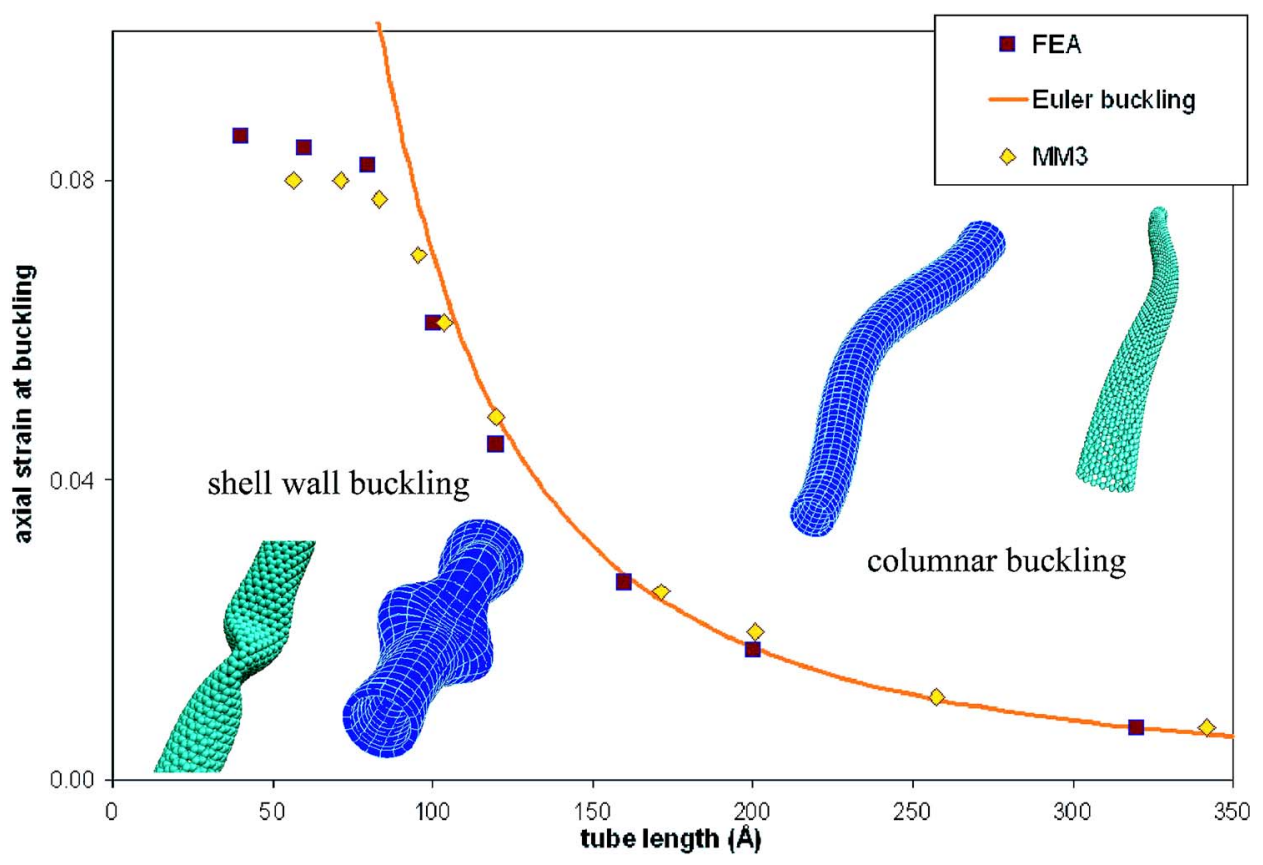

FIG. 12. (Color online) Axial strain at buckling vs tube length for a $(16,0)$ SWNT (tube diameter $=11.9 \AA$ ) computed from MM simulations, FE analysis and the Euler buckling theory. 


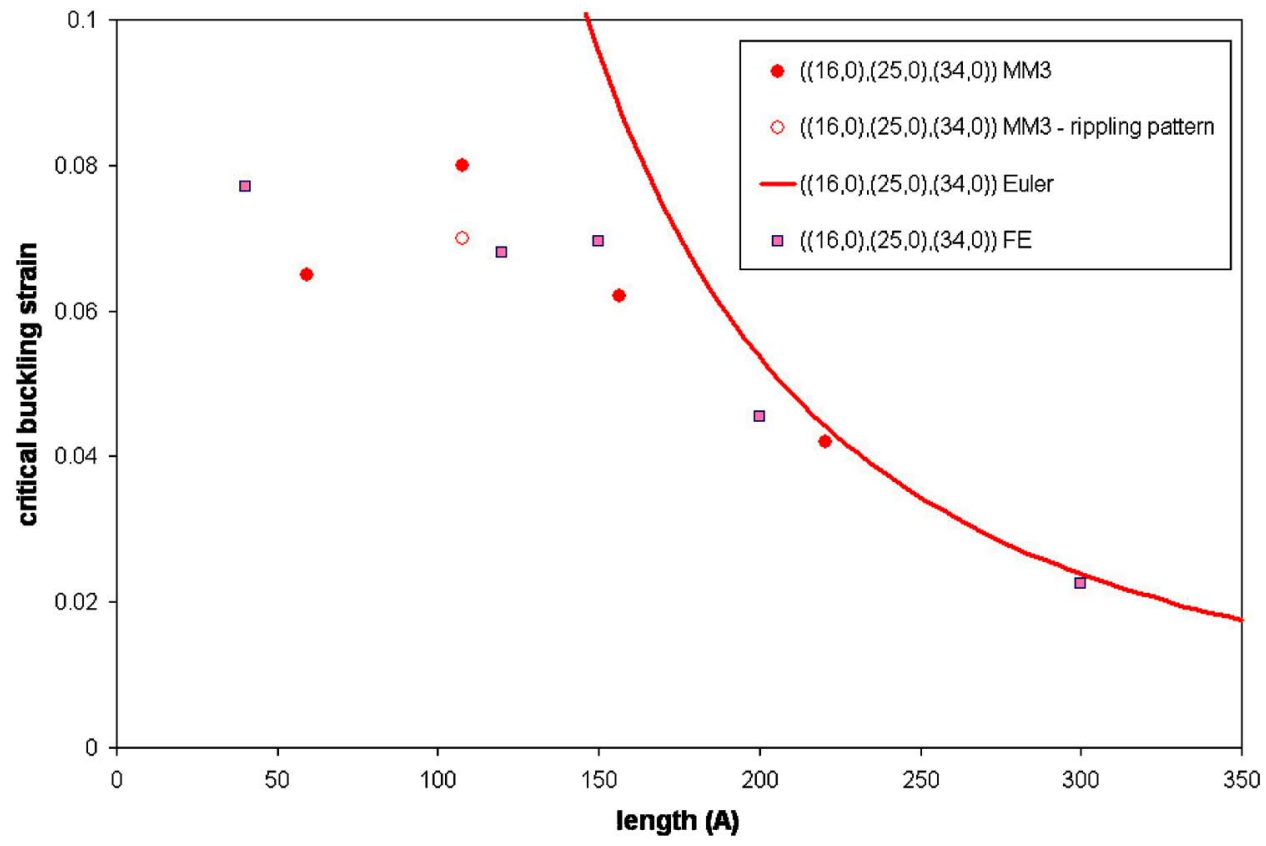

FIG. 13. (Color online) For a DWNT, axial strain at the initiation of buckling vs the tube length from FE analyses of five continuum structures (analyses with Euler buckling theory for three continuum structures that differ in $E$ and wall thicknesses, and two different ways of modeling van der Waals forces in the FE analysis) and the MM simulations. were studied with the MM simulations, and of their equivalent continuum structures by the FE method and the Euler buckling theory on the assumption that the two end faces are clamped. A sinusoidal perturbation with amplitude of $0.5 \%$ of the tube length, introduced to facilitate buckling in the MM simulations, was also applied in the FE analyses.

Results from the Euler buckling theory for the following three continuum structures equivalent to the DWNT were computed: (a) two concentric tubes of mean diameter equal to that of the walls of the DWNT, $h=3.4 \AA, E=0.99 \mathrm{TPa}$; (b) same as in (a) except $h=1.34 \AA, E=2.53 \mathrm{TPa}$; (c) each tube modeled as a thin shell of thickness $0.66 \AA, E=5.12 \mathrm{TPa}$. Thus, the equivalent stiffness $E A / L$ for the three continuum structures is the same. For the FE analysis, the DWNT was replaced by the continuum structure (b). The nodes on these surfaces were connected by truss elements whose axial force versus axial strain curve is shown in Fig. 4 for both the pseudopressure and the trilinear cases.

Results from these studies are exhibited in Fig. 13. We note that the Euler column buckling predictions match well with those from the MM simulations. In the columnar buckling regime, the FE analysis of each one of the three continuum structures gives results close to those obtained from the MM simulations. The FE analyses could also predict the transition of buckling from columnar to shell wall mode. It shows that it is less critical how van der Waals forces are modeled in the continuum structure as far as the analysis of columnar buckling and its transition into shell wall buckling mode is concerned. In fact, the trilinear truss model seems to perform slightly better, especially in the pure shell wall buckling modes. With the radial pressure versus the radial strain relation fitted to MM simulations, the computed axial strain at buckling is slightly higher than that given by the MM simulations.

For the DWNT studied, buckling modes obtained from the MM simulations and the FE analyses of the two continuum structures are depicted in Fig. 14. Mode shapes for the pure shell wall buckling and columnar buckling modes match well with those obtained from the MM simulations. However, mixed modes during the transition from columnar to shell wall buckling were more difficult to capture; it is due to the simplicity of continuum models used here. The continuum model of Arroyo and Belytschko ${ }^{21}$ predicts some of complex buckling modes but it is considerably more sophisticated than the ones examined here.

Buckling deformations of a continuum structure equivalent to a TWNT were also analyzed and results were found to be similar to those for a DWNT; these are shown in Fig. 15. The van der Waals forces were modeled with the same approaches as those used for continuum structures equivalent to the DWNTs. As for the DWNT, the FE analysis of deformations of the continuum structure can predict well the columnar buckling mode. Results for the transition from the columnar to the shell wall buckling mode are qualitatively similar to those obtained from the MM simulations. How-

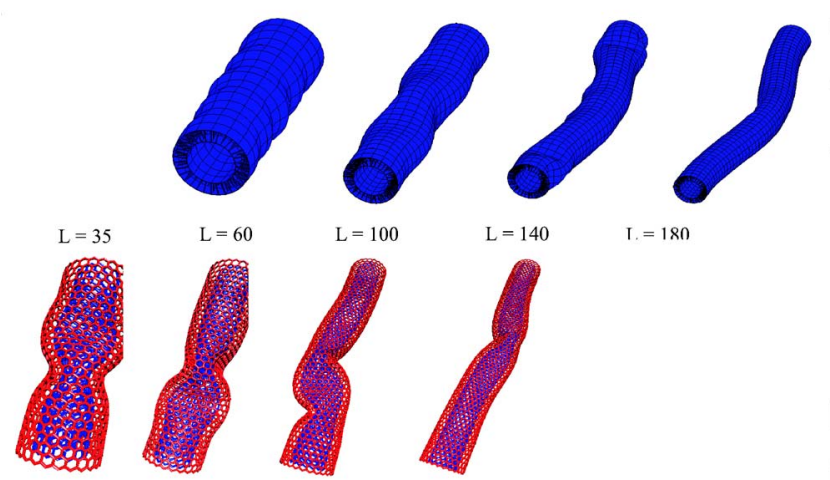

FIG. 14. (Color online) For a DWNT, buckling modes computed with (top) FE analyses of equivalent continuum structures with truss elements connecting corresponding atoms of the two tubes, and (bottom) MM simulations; pictures in the bottom part are shifted to the left to align them with proper values of their lengths. 


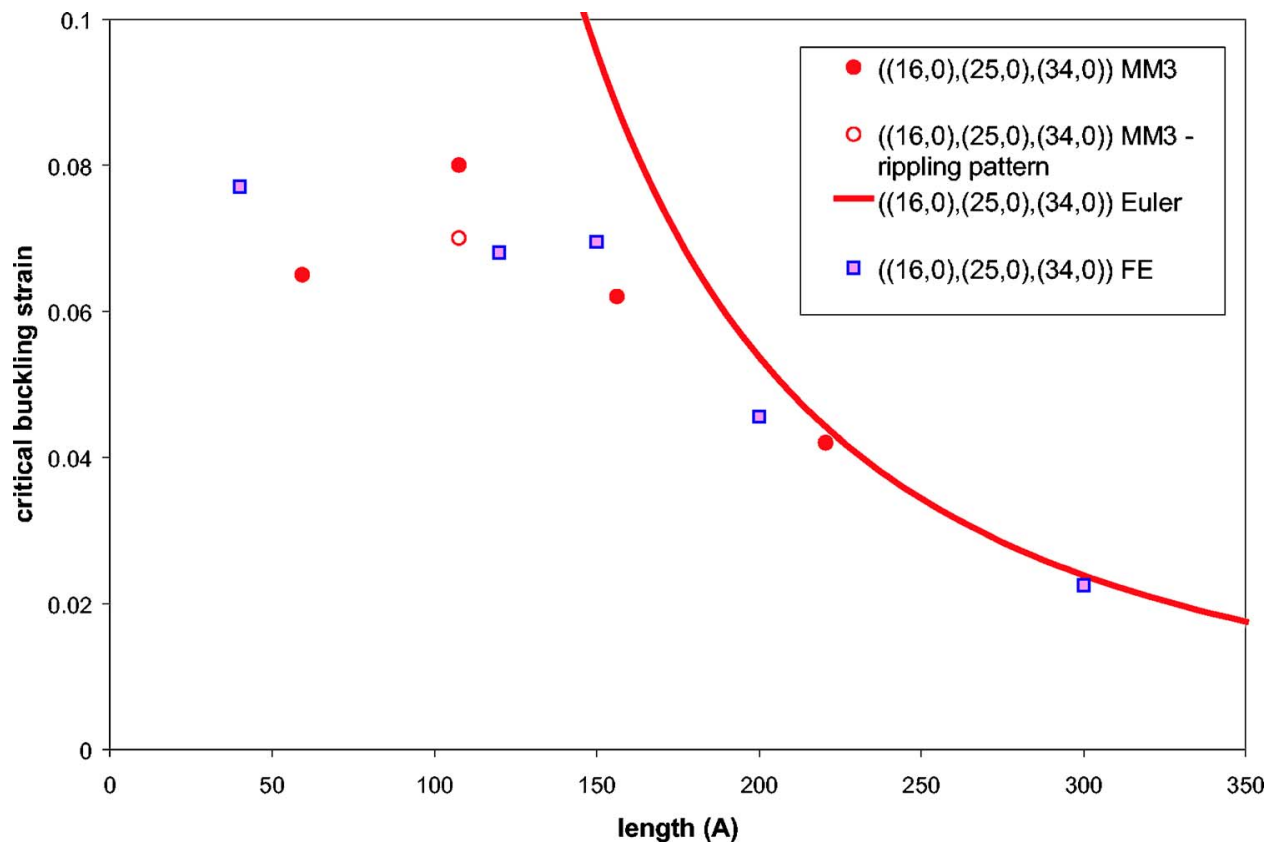

FIG. 15. (Color online) For a TWNT, axial strain at buckling vs tube length computed from the FE analysis of equivalent continuum structures and MM simulations.

ever, the FE analysis was not able to replicate all of the buckling modes found from the MM simulations. The open circle in the figure indicates when rippling deformation modes, exhibited in Fig. 10, appeared during axial compression of the TWNT. This deformation mode is neither predicted by the FE analysis nor by the Euler buckling theory. There is no experimental data available on buckling deformations of a MWNT; thus computed results could not be compared with experimental observations.

\section{CONCLUSIONS}

We have studied buckling of axially compressed multiwalled carbon nanotubes by using molecular mechanics simulations, the Euler buckling theory, and the finite element method; the latter two approaches employ continuum structures equivalent to the nanotubes. It is found that the van der Waals forces among atoms on adjacent walls of multiwalled nanotubes play a significant role. The van der Waals forces cause the MWNT to generally buckle as a unit, that is, the buckled shape is the same for all walls. The present work makes the following new contributions.

For columnar buckling modes, the axial strain at the onset of buckling equaled that given by the simple Euler column theory. The buckling mode transitions from columnar to shell wall buckling as the tube length is decreased. During the transition columnar buckling modes include crimping which is a form of shell wall buckling. The axial strain at the ini- tiation of the shell wall buckling mode equals the largest of those of the single-walled nanotubes constituting a multiwalled nanotube. An interesting rippling pattern was found in the buckling response of a triple-walled nanotube which illustrates the resistance provided by the individual walls against buckling.

The buckling modes computed from the finite element analysis of equivalent continuum structures, with the van der Waals forces simulated by truss elements, are close to those obtained from the molecular mechanics simulations; however, the transition zone mode shapes are not replicated. Finite element results suggest that while the consideration of the van der Waals forces is critical, their exact modeling is not very critical as adequate results were found for two different mathematical representations. The modeling of van der Waals forces by truss elements is new and provides an easy way to simulate them. It helps understand how these forces coordinate the buckling of different walls of a nanotube. The magnitude of the van der Waals force can be computed from Eq. (1).

\section{ACKNOWLEDGMENTS}

This work was partially supported by the ONR Grant No. N0014-98-1-0300 to Virginia Polytechnic Institute and State University with Dr. Y. D. S. Rajapakse as the program manager, and by the AFOSR MURI subcontract from Georgia Institute of Technology.
${ }^{1}$ P. Poncharal, Z. L. Wang, D. Ugarte, and W. A. de Heer, Science 283, 5407 (1999).

${ }^{2}$ C. Bower, R. Rosen, L. Jin, J. Han, and O. Zhou, Appl. Phys. Lett. 74, 3317 (1999).
${ }^{3}$ O. Lourie, D. M. Cox, and H. D. Wagner, Phys. Rev. Lett. 81, 1638 (1998).

${ }^{4}$ M. R. Falvo, G. J. Clary, R. M. Taylor II, V. Chi, F. P. Brooks, Jr., S. Washburn, and R. Superfine, Nature (London) 389, 582 
(1997).

${ }^{5}$ R. S. Ruoff and D. C. Lorents, Carbon 33, 925 (1995).

${ }^{6}$ J. F. Despres, D. Daguerre, and K. Lafdi, Carbon 33, 87 (1995).

${ }^{7}$ K. M. Liew, C. H. Wong, X. Q. He, M. J. Tan, and S. A. Meguid, Phys. Rev. B 69, 115429 (2004).

${ }^{8}$ M.-F. Yu, B. I. Yakobson, and R. S. Ruoff, J. Phys. Chem. B 104, 8764 (2000).

${ }^{9}$ J. Cunnings and A. Zettl, Science 289, 602 (2000).

${ }^{10}$ O. Lourie, D. M. Cox, and H. D. Wagner, Phys. Rev. Lett. 81, 1638 (1998).

${ }^{11}$ C. Bower, R. Rosen, L. Jin, J. Han, and O. Zhou, Appl. Phys. Lett. 74, 3317 (1999)

${ }^{12}$ M. R. Falvo, G. J. Clary, R. M. Taylor, V. Chi, F. P. Brooks, S. Washburn, and R. Superfine, Nature (London) 389, 582 (1997).

${ }^{13}$ M. F. Yu, O. Lourie, M. J. Dyer, K. Moloni, T. F. Kelly, and R. S. Ruoff, Science 287, 637 (2000).

${ }^{14}$ E. T. Thostenson and T.-W. Chou, Carbon 42, 3003 (2004).

${ }^{15}$ D. Troya, S. L. Mielke, and G. C. Schatz, Chem. Phys. Lett. 382, 133 (2003).

${ }^{16}$ B. I. Yakobson, C. J. Brabec, and J. Bernholc, Phys. Rev. Lett. 76, 2511 (1996).

${ }^{17}$ A. Sears and R. C. Batra, Phys. Rev. B 69, 235406 (2004).

${ }^{18}$ C. Q. Ru, J. Appl. Phys. 87, 7227 (2000).

${ }^{19}$ A. Pantano, M. C. Boyce, and D. M. Parks, Phys. Rev. Lett. 91 145504 (2003).

${ }^{20}$ A. Pantano, M. C. Boyce, and D. M. Parks, J. Eng. Mater.
Technol. 126, 279 (2004).

${ }^{21}$ M. Arroyo and T. Belytschko, Int. J. Numer. Methods Eng. 59, 419 (2003).

${ }^{22}$ C. T. White, D. H. Robertson, and J. W. Mintmire, Phys. Rev. B 47, 5485 (1992).

${ }^{23}$ A.-J. C. B. Saint-Venant, Memoires des Savants Etrangers 14, 233 (1856).

${ }^{24}$ P. Poncharal, Z. L. Wang, D. Ugarte, and W. A. de Heer, Science 283, 1513 (1999).

${ }^{25}$ Z. L. Wang, P. Poncharal, W. A. de Heer, J. Phys. Chem. Solids 61, 1025 (2000).

${ }^{26}$ M. F. Yu, O. Lourie, M. J. Dyer, K. Moloni, T. F. Keely, and R. S. Ruoff, Science 287, 637 (2000).

${ }^{27}$ E. W. Wong, P. E. Sheehan, and C. M. Lieber, Science 277, 1971 (1997).

${ }^{28}$ H. J. Qi, K. B. Teo, K. Lqu, M. C. Boyce, W. I. Milne, J. Robertson, and K. K. Gleason, J. Mech. Phys. Solids 51, 2213 (2003).

${ }^{29}$ W. F. Riley, L. D. Sturges, and D. H. Morris, Mechanics of Materials (Wiley, New York, 1999), Chap. 9.

${ }^{30}$ N. L. Allinger, Y. H. Yuh, and J.-H. Lii, J. Am. Chem. Soc. 111, 8551 (1989).

${ }^{31}$ J. W. Ponder (private communication).

${ }^{32}$ D. S. Cairns, J. F. Mandell, A. Sears, and L. R. McKittrick (unpublished).

${ }^{33}$ R. C. Batra and A. Sears (unpublished). 